\title{
Abstraction for Falsification
}

\author{
Thomas Ball ${ }^{1}$, Orna Kupferman ${ }^{2}$, and Greta Yorsh ${ }^{3}$ \\ ${ }^{1}$ Microsoft Research, Redmond, WA, USA \\ tball@microsoft.com \\ www.research.microsoft. com/ tball \\ ${ }^{2}$ Hebrew University, School of Eng. and Comp. Sci., Jerusalem 91904, Israel \\ orna@cs.huji.ac.il \\ www.cs.huji.ac.il/ orna \\ 3 Tel-Aviv University, School of Comp. Sci., Tel-Aviv 69978, Israel \\ gretaylpost.tau.ac.il \\ www. math.tau.ac.il/ gretay
}

\begin{abstract}
Abstraction is traditionally used in the process of verification. There, an abstraction of a concrete system is sound if properties of the abstract system also hold in the concrete system. Specifically, if an abstract state $a$ satisfies a property $\psi$ then all the concrete states that correspond to $a$ satisfy $\psi$ too. Since the ideal goal of proving a system correct involves many obstacles, the primary use of formal methods nowadays is falsification. There, as in testing, the goal is to detect errors, rather than to prove correctness. In the falsification setting, we can say that an abstraction is sound if errors of the abstract system exist also in the concrete system. Specifically, if an abstract state $a$ violates a property $\psi$, then there exists a concrete state that corresponds to $a$ and violates $\psi$ too.

An abstraction that is sound for falsification need not be sound for verification. This suggests that existing frameworks for abstraction for verification may be too restrictive when used for falsification, and that a new framework is needed in order to take advantage of the weaker definition of soundness in the falsification setting.

We present such a framework, show that it is indeed stronger (than other abstraction frameworks designed for verification), demonstrate that it can be made even stronger by parameterizing its transitions by predicates, and describe how it can be used for falsification of branching-time and linear-time temporal properties, as well as for generating testing goals for a concrete system by reasoning about its abstraction.
\end{abstract}

\section{Introduction}

Automated abstraction is a powerful technique for reasoning about systems. An abstraction framework [CC77] consists of a concrete system with (large, possibly infinite) state space $C$, an abstract system with (smaller, often finite) state space $A$, and an abstraction function $\rho: C \rightarrow A$ that relates concrete and abstract states. An abstraction framework is sound with respect to a logic $L$ if all properties specified in $L$ that hold in an abstract state $a$ also hold in all the concrete states that correspond to $a$. Formally, for all $a \in A$ and $\varphi \in L$, if $a$ satisfies $\varphi$ then for all $c \in C$ with $\rho(c, a)$, we have that $c$ satisfies $\varphi$. 
The soundness of the abstraction framework enables the user to verify properties of the abstract system using techniques such as model checking [CE81, QS81] and conclude their validity in the concrete system.

While the ultimate goal of formal verification is to prove that a system satisfies some specification, there are many obstacles to achieving this ideal in practice. Thus, the primary use of formal methods nowadays is falsification, where the goal is to detect errors rather than to provide a proof of correctness. This is reflected in the extensive research done on bounded model checking (c.f., $\left[\mathrm{FKZ}^{+} 00\right]$ ), runtime verification (c.f., [Sip99]), etc. In the falsification setting, we can say that an abstraction is sound with respect to a logic $L$ if all errors specified in $L$ that hold in an abstract state $a$ also hold in some concrete state that corresponds to $a$. Formally, for all $a \in A$ and $\varphi \in L$, if $a$ satisfies $\varphi$ then there is $c \in C$ such that $\rho(c, a)$ and $c$ satisfies $\varphi$. ${ }^{1}$ Since every abstract state corresponds to at least one concrete state, the soundness condition in the falsification setting is weaker than the soundness condition in the verification setting. To see that this weaker definition is sufficiently strong for falsification, note that the concrete state $c$ that satisfies $\varphi$ witnesses that the concrete system is erroneous (we note that in the falsification setting $\varphi$ is a "bad" property that we don't wish the system to have, while in the verifications setting $\varphi$ is a "good" property that we wish the system to have).

We develop a new abstraction framework to take advantage of the weaker definition of soundness in the falsification setting. Our framework is based on modal transition systems (MTS) [LT88]. Traditional MTS have two types of transitions: may (over-approximating transitions) and must (under-approximating transitions). The use of must transitions in the falsification setting was explored in [PDV01, GLST05], with different motivations. Our framework contains, in addition, a new type of transition, which can be viewed as the reverse version of must transitions [Bal04]. Accordingly, we refer to transitions of this type as must $^{-}$transitions and refer to the traditional must transitions as must $^{+}$transitions. While a must $^{+}$transition from an abstract state $a$ to an abstract state $a^{\prime}$ implies that for all concrete states $c$ with $\rho(c, a)$ there is a successor concrete state $c^{\prime}$ with $\rho\left(c^{\prime}, a^{\prime}\right)$, a must ${ }^{-}$transition from $a$ to $a^{\prime}$ implies that for all concrete states $c^{\prime}$ with $\rho\left(c^{\prime}, a^{\prime}\right)$ there is a concrete predecessor state $c$ with $\rho(c, a)$. The must ${ }^{-}$transitions correspond to the weaker soundness requirement in the falsification setting and are incomparable to must $^{+}$transitions.

Consider, for example, a simple concrete system consisting of the assignment statement $\mathrm{x}:=\mathrm{x}-3$. Suppose that the abstract system is formed via predicate abstraction using the predicate $x>6$. Consider the abstract transition $\{x>6\} \mathrm{x}:=\mathrm{x}-3\{x>6\}$. This transition is not a must transition, as there are pre-states satisfying $x>6$ (namely $x=7, x=8$, and $x=9$ ) for which the assignment statement results in a poststate that does not satisfy $x>6$. Therefore, in a traditional MTS this transition is a

\footnotetext{
${ }^{1}$ Note that the falsification setting is different than the problem of generalized model checking [GJ02]. There, the existential quantifier ranges over all possible concrete systems and the problem is one of satisfiability (does there exist a concrete system with the same property as the abstract system?). Here, the concrete system is given and we only replace the universal quantification on concrete states that correspond to $a$ by an existential quantification on them.
} 
may transition. However, in an MTS with must $^{-}$transitions, the above transition is a must ${ }^{-}$transition, as for every post-state $c^{\prime}$ satisfying $x>6$ there is a pre-state $c$ satisfying $x>6$ such that the execution of $\mathrm{x}:=\mathrm{x}-3$ from $c$ yields $c^{\prime}$. It is impossible to make this inference in a traditional MTS, even those augmented with hyper-must transitions [LX90, SG04]. As we shall see below, the observation that the abstract transition is a must $^{-}$transition rather than a may transition enables better reasoning about the concrete system.

We study MTS with these three types of transitions, which we refer to as ternary modal transition systems (TMTS) ${ }^{2}$. We first show that the TMTS model is indeed stronger than the MTS model: while MTS with only may and must $^{+}$transitions are logically characterized by a 3 -valued modal logic with the $A X$ and $E X$ (for all successors/exists a successor) operators, TMTS are logically characterized by a strictly more expressive modal logic which has, in addition, the $A Y$ and $E Y$ (for all predecessors/exists a predecessor) past operators. We then show that by replacing must $^{+}$ transitions by must $^{-}$transitions, existing work on abstraction/refinement for verification [GHJ01, SG03, BG04, SG04, DN05] can be lifted to abstraction/refinement for falsification.

In particular, this immediately provides a framework for falsification of CTL and $\mu$ calculus specifications. Going back to our example, by letting existential quantification range over must $^{-}$transitions, we can conclude from the fact that the abstract system satisfies the property $E X x>6$ (there is a successor in which $x>6$ is valid) that some concrete state also satisfies $E X x>6$. Note that such reasoning cannot be done in a traditional MTS, as there the must $^{-}$transition is overapproximated by a may transition, which is not helpful for reasoning about existential properties. Thus, there are cases where evaluation of a formula on a traditional MTS returns $\perp$ (nothing can be concluded for the concrete system, and refinement is needed) and its evaluation on a TMTS returns an existential true or existential false. Formally, we describe a 6-valued falsification semantics for TMTS. In addition to the $\mathbf{T}$ (all corresponding concrete states satisfy the formula), $\mathbf{F}$ (all corresponding concrete states violate the formula), and $\perp$ truth values that the 3-valued semantics for MTS has, the falsification semantics also has the $\mathbf{T}_{\exists}$ (there is a corresponding concrete state that satisfies the formula), $\mathbf{F}_{\exists}$ (there is a corresponding concrete state that violates the formula), and $M$ (mixed - both $\mathbf{T}_{\exists}$ and $\mathbf{F}_{\exists}$ hold) truth values.

The combination of must $^{+}$and must $^{-}$transitions turn out to be especially powerful when reasoning about weak reachability, which is useful for abstraction-guided test generation [Bal04] and falsification of linear-time properties. As discussed in [Bal04], if there is a sequence of must $^{-}$transitions from $a_{0}$ to $a_{j}$ followed by a sequence of must ${ }^{+}$transitions from $a_{j}$ to $a_{k}$, then there are guaranteed to be concrete states $c_{0}$ and $c_{k}$ (corresponding to $a_{0}$ and $a_{k}$ ) such that $c_{k}$ is reachable from $c_{0}$ in the concrete system (in which case we say that $a_{k}$ is weakly reachable from $a_{0}$ ). In this case, we can conclude that it is possible to cover the abstract state $a_{k}$ via testing. When the abstraction is the product of an abstract system with a nondeterministic Büchi automaton accepting all the faults of the system, weak reachability can be used in order to de-

\footnotetext{
${ }^{2}$ Not to be confused with the three-valued logic sometimes used in these systems.
} 
tect faults in the concrete system. We focus on abstractions obtained from programs by predicate abstraction, and study the problem of composing transitions in an TMTS in a way that guarantees weak reachability. We suggest a method where must $^{+}$and must $^{-}$ transitions are parameterized with predicates, automatically induced by the weakest preconditions and the strongest postconditions of the statements in the program ${ }^{3}$.

The paper is organized as follows. Section 2 formally presents ternary modal transition systems (TMTS), how they abstract concrete systems (as well as each other) and characterizes their abstraction pre-order via the full propositional modal logic (fullPML). Section 2.3 presents the 6 -valued falsification semantics for TMTS and demonstrates that TMTS are more precise for falsification than traditional MTS. We also show that falsification can be lifted to the $\mu$-calculus as well as linear-time logics. Section 3 shows that weak reachability can be made more precise by parameterizing both must $^{+}$ and must $^{-}$transitions via predicates. Section 4 describes describes applications of TMTS to abstraction-guided testing and to model checking. Section 5 concludes the paper.

Due to a lack of space, this version does not contain proofs and contains only a partial discussion of the results. For a full version, the reader is referred to the authors' URLs and our technical report [BKY05].

\section{The Abstraction Framework}

In this section we describe our abstraction framework. We define TMTS - ternary modal transition systems, which extend modal transition systems by a third type of transition, and study their theoretical aspects.

\subsection{Ternary Modal Transition Systems}

A concrete transition system is a tuple $C=\left\langle A P, S_{C}, I_{C}, \longrightarrow_{C}, L_{C}\right\rangle$, where $A P$ is a finite set of atomic propositions, $S_{C}$ is a (possibly infinite) set of states, $I_{C} \subseteq S_{C}$ is a set of initial states, $\longrightarrow C \subseteq S_{C} \times S_{C}$ is a transition relation and $L_{C}: S_{C} \times A P \mapsto\{\mathbf{T}, \mathbf{F}\}$ is a labeling function that maps each state and atomic proposition to the truth value of the proposition in the state. ${ }^{4}$

An abstraction of $C$ is a partially defined system. Incompleteness involves both the value of the atomic propositions, which can now take the value $\perp$ (unknown), and the transition relation, which is approximated by over- and/or under-approximating transitions. Several frameworks are defined in the literature (c.f. [LT88, BG99, HJS01]). We define here a new framework, which consists of ternary transition systems (TMTS, for short). Unlike the traditional MTS, our TMTS has two types of under-approximating transitions. Formally, we have the following.

\footnotetext{
${ }^{3}$ We note (see the remark at the end of Section 3 for a detailed discussion) that our approach is different than refining the TMTS as the predicates we use are local to the transitions.

${ }^{4}$ We use $\mathbf{T}$ and $\mathbf{F}$ to denote the truth values true and false of the standard (verification) semantics, and introduce additional truth values in Section 2.3.
} 
A TMTS is a tuple $A=\left\langle A P, S_{A}, I_{A}, \stackrel{\text { may }}{\longrightarrow} A, \stackrel{m u s t}{+}_{A}, \stackrel{m u s t}{-}_{A}, L_{A}\right\rangle$, where $A P$ is a finite set of atomic propositions, $S_{A}$ is a finite set of abstract states, $I_{A} \subseteq S_{A}$ is a set of initial states, the transition relations $\stackrel{\text { may }}{\longrightarrow}_{A}, \stackrel{\text { must }}{+}_{A}$, and $\stackrel{\text { must }}{ }_{A}^{-}$are subsets of $S_{A} \times S_{A}$ satisfying $\stackrel{\text { must }}{+}_{A} \subseteq \stackrel{\text { may }}{\longrightarrow}_{A}$ and $\stackrel{\text { must }}{ }_{A} \subseteq \stackrel{\text { may }}{\longrightarrow}_{A}$, and $L_{A}: S_{A} \times A P \rightarrow\{\mathbf{T}, \mathbf{F}, \perp\}$ is a labeling function that maps each state and atomic proposition to the truth value (possibly unknown) of the proposition in the state. When $A$ is clear from the context we sometimes use may $\left(a, a^{\prime}\right)$, must $^{+}\left(a, a^{\prime}\right)$, and must $^{-}\left(a, a^{\prime}\right)$ instead of $a \stackrel{\text { may }}{\longrightarrow} a, a \stackrel{m_{u s t}^{+}}{\longrightarrow} a^{\prime}$, and $a \stackrel{\text { must }}{\longrightarrow} a^{\prime}$, respectively.

The elements of $\{\mathbf{T}, \mathbf{F}, \perp\}$ can be arranged in an "information lattice" [Kle87] in which $\perp \sqsubseteq \mathbf{T}$ and $\perp \sqsubseteq \mathbf{F}$. We say that a concrete state $c$ satisfies an abstract state $a$ if for all $p \in A P$, we have $L_{A}(a, p) \sqsubseteq L_{C}(c, p)$ (equivalently, if $L_{A}(a, p) \neq \perp$ then $\left.L_{C}(c, p)=L_{A}(a, p)\right)$.

Let $C=\left\langle A P, S_{C}, I_{C}, \longrightarrow_{C}, L_{C}\right\rangle$ be a concrete transition system. A TMTS $A=$ $\left\langle A P, S_{A}, I_{A}, \stackrel{\text { may }}{\longrightarrow}, \stackrel{\text { must }^{+}}{\longrightarrow}, \stackrel{\text { must }^{-}}{\longrightarrow}, L_{A}\right\rangle$ is an abstraction of $C$ if there exists a total and onto function $\rho: S_{C} \rightarrow S_{A}$ such that (i) for all $c \in S_{C}$, we have that $c$ satisfies $\rho(c)$, and (ii) the transition relations $\stackrel{\text { may }}{\longrightarrow}_{A}, \stackrel{\text { must }}{ }_{A}$, and $\stackrel{\text { must }}{ }_{A}^{-}$satisfy the following:

- $a \stackrel{\text { may }}{\longrightarrow} a^{\prime}$ if there is a concrete state $c$ with $\rho(c)=a$, there is a concrete state $c^{\prime}$ with $\rho\left(c^{\prime}\right)=a^{\prime}$, and $c \longrightarrow C c^{\prime}$.

- $a \stackrel{\text { must }}{\longrightarrow} A a^{\prime}$ only if for every concrete state $c$ with $\rho(c)=a$, there is a concrete state $c^{\prime}$ with $\rho\left(c^{\prime}\right)=a^{\prime}$ and $c \longrightarrow C c^{\prime}$.

- $a \stackrel{\text { must }}{\longrightarrow}_{A} a^{\prime}$ only if for every concrete state $c^{\prime}$ with $\rho\left(c^{\prime}\right)=a^{\prime}$, there is a concrete state $c$ with $\rho(c)=a$ and $c \longrightarrow C c^{\prime}$.

Note that may transitions over-approximate the concrete transitions. In particular, the abstract system can contain may transitions for which there is no corresponding concrete transition. Dually, must ${ }^{-}$and must $^{+}$transitions under-approximate the concrete transitions. Thus, the concrete transition relation can contain transitions for which there are no corresponding must transitions. Since $\rho$ is onto, each abstract state corresponds to at least one concrete state, and so $\stackrel{m u s t}{\longrightarrow}_{A} \subseteq \stackrel{\text { may }}{\longrightarrow} A$ and $\stackrel{\text { must }}{\longrightarrow}_{A} \subseteq \stackrel{\text { may }}{\longrightarrow}_{A}$. On the other hand, $\stackrel{\text { must }^{+}}{\longrightarrow} A$ and $\stackrel{\text { must }}{\longrightarrow}_{A}$ are incomparable. Finally, note that by letting musttransitions become may-transitions, and by adding superfluous may-transitions, we can have several abstractions of the same concrete system.

A precision preorder on TMTS defines when one TMTS is more abstract than another. For two TMTS $A=\left\langle A P, S_{A}, I_{A}, \stackrel{\text { may }}{\longrightarrow}_{A}, \stackrel{m u s t}{+}_{A}, \stackrel{m u s t}{-}_{A}, L_{A}\right\rangle$ and $B=\left\langle A P, S_{B}\right.$, $\left.I_{B}, \stackrel{m a y}{\longrightarrow}, \stackrel{m^{\prime} t^{+}}{\longrightarrow}, \stackrel{m u s t^{-}}{\longrightarrow}, L_{B}\right\rangle$, the precision preorder is the greatest relation $\mathcal{H} \subseteq S_{A} \times$ $S_{B}$ such that if $\mathcal{H}(a, b)$ then

C0. for all $p \in A P$, we have $L_{A}(a, p) \sqsubseteq L_{B}(b, p)$,

C1. if $b \stackrel{\text { may }}{\longrightarrow}_{B} b^{\prime}$, then there is $a^{\prime} \in S_{A}$ such that $\mathcal{H}\left(a^{\prime}, b^{\prime}\right)$ and $a \stackrel{\operatorname{may}}{\longrightarrow}_{A} a^{\prime}$,

C2. if $b^{\prime} \stackrel{\text { may }}{\longrightarrow}_{B} b$, then there is $a^{\prime} \in S_{A}$ such that $\mathcal{H}\left(a^{\prime}, b^{\prime}\right)$ and $a^{\prime} \stackrel{\text { may }}{\longrightarrow}_{A} a$, 
C3. if $a \stackrel{\text { must }}{\longrightarrow} A a^{\prime}$, then there is $b^{\prime} \in S_{B}$ such that $\mathcal{H}\left(a^{\prime}, b^{\prime}\right)$ and $b \stackrel{\text { must }}{\longrightarrow} B$ C4. if $a^{\prime} \stackrel{\text { must }^{-}}{\longrightarrow} a$, then there is $b^{\prime} \in S_{B}$ such that $\mathcal{H}\left(a^{\prime}, b^{\prime}\right)$ and $b^{\prime} \stackrel{\text { must }}{-}_{B} b$.

When $\mathcal{H}(a, b)$, we write $(A, a) \preceq(B, b)$, which indicates that $A$ is more abstract (less defined) than $B$.

By viewing a concrete system as an abstract system whose may, ust $^{+}$, and must $^{-}$ transition relations are equivalent to the transition relation of the concrete system, we can use the precision preorder to relate a concrete system and its abstraction. Formally, the precision preorder $\mathcal{H} \subseteq S_{C} \times S_{A}$ (also known as mixed simulation [DGG97, GJ02]) is such that $\mathcal{H}(c, a)$ iff $\rho(c)=a$.

\subsection{A Logical Characterization}

The logic full-PML is a propositional logic extended with the modal operators $A X$ ("for all immediate successors") and $A Y$ ("for all immediate predecessors"). Thus, full-PML extends PML [Ben91] by the past-time operator $A Y$. The syntax of full-PML is given by the rules $\theta::=p|\neg \theta| \theta \wedge \theta|A X \theta| A Y \theta$, for $p \in A P$.

We define a 3-valued semantics of full-PML formulas with respect to TMTS. The value of a formula $\theta$ in a state $a$ of a TMTS $A=\left\langle S_{A}, I_{A}, \stackrel{\text { may }}{\longrightarrow}, \stackrel{\text { must }}{\longrightarrow} A, \stackrel{\text { must }}{\longrightarrow}_{A}, L_{A}\right\rangle$, denoted $[(A, a) \models \theta]$, is defined as follows:

$$
\begin{aligned}
& {[(A, a) \models p]=L_{A}(a, p) .} \\
& {[(A, a) \models \neg \theta]=\left\{\begin{array}{l}
\mathbf{T} \text { if }[(A, a) \models \theta]=\mathbf{F} . \\
\mathbf{F} \text { if }[(A, a) \models \theta]=\mathbf{T} . \\
\perp \text { otherwise. }
\end{array}\right.} \\
& {\left[(A, a) \models \theta_{1} \wedge \theta_{2}\right]=\left\{\begin{array}{l}
\mathbf{T} \text { if }\left[(A, a)=\theta_{1}\right]=\mathbf{T} \text { and }\left[(A, a) \models \theta_{2}\right]=\mathbf{T} . \\
\mathbf{F} \text { if }\left[(A, a)=\theta_{1}\right]=\mathbf{F} \text { or }\left[(A, a) \models \theta_{2}\right]=\mathbf{F} . \\
\perp \text { otherwise. }
\end{array}\right.} \\
& {[(A, a) \models A X \theta]= \begin{cases}\mathbf{T} \text { if for all } a^{\prime}, \text { if } \operatorname{may}\left(a, a^{\prime}\right) \text { then }\left[\left(A, a^{\prime}\right) \models \theta\right]=\mathbf{T} . \\
\mathbf{F} \text { if exists } a^{\prime} \text { s.t. } \operatorname{must}\left(a, a^{\prime}\right) \text { and }\left[\left(A, a^{\prime}\right) \models \theta\right]=\mathbf{F} . \\
\perp \text { otherwise. }\end{cases} } \\
& {[(A, a) \models A Y \theta]= \begin{cases}\mathbf{T} \text { if for all } a^{\prime} \text {, if } \operatorname{may}\left(a^{\prime}, a\right) \text { then }\left[\left(A, a^{\prime}\right) \models \theta\right]=\mathbf{T} . \\
\mathbf{F} \text { if exists } a^{\prime} \text { s.t. } \operatorname{must}\left(a^{\prime}, a\right) \text { and }\left[\left(A, a^{\prime}\right) \models \theta\right]=\mathbf{F} . \\
\perp \text { otherwise. }\end{cases} }
\end{aligned}
$$

While PML logically characterizes the precision preorder on MTS [GJ02], fullPML characterizes the precision preorder on TMTS. It follows that the TMTS model is indeed stronger than the MTS model, because TMTS are logically characterized by a strictly more expressive modal logic which has the past operators $A Y$ and $E Y$, in addition to $A X$ and $E X$ operators. Formally, we have the following.

Theorem 1. Let $A=\left\langle A P, S_{A}, I_{A}, \stackrel{\text { may }}{\longrightarrow}, \stackrel{\text { must }^{+}}{\rightarrow}, \stackrel{\text { must }}{-}_{A}, L_{A}\right\rangle$ and $B=\left\langle A P, S_{B}, I_{B}\right.$, $\left.\stackrel{\text { may }}{\longrightarrow}_{B}, \stackrel{\text { must }^{+}}{\longrightarrow}, \stackrel{\text { must }^{-}}{\longrightarrow}, L_{B}\right\rangle$ be two TMTS. For every two states $a \in S_{A}$ and $b \in S_{B}$, we have that $(A, a) \preceq(B, b)$ iff $[(A, a) \models \theta] \sqsubseteq[(B, b) \models \theta]$ for all full-PML formulas $\theta$. 


\subsection{Falsification Using TMTS}

As shown in Section 2.2, the backwards nature of must $^{-}$transitions makes them suitable for reasoning about the past. Thus, TMTS can be helpful in the verification setting for reasoning about specifications in full $\mu$-calculus and other specification formalisms that contain past operators. We view this as a minor advantage of TMTS. In this section we study their significant advantage: reasoning about specifications in a falsification setting ${ }^{5}$.

In addition to the truth values $\mathbf{T}, \mathbf{F}$, and $\perp$, we now allow formulas to have the values $\mathbf{T}_{\exists}$ (existential true), $\mathbf{F}_{\exists}$ (existential false), and $\mathbf{M}$ ("mixed" - both $\mathbf{T}$ and $\mathbf{F}$ ). Intuitively, the values $\mathbf{T}_{\exists}, \mathbf{F}_{\exists}$, and $\mathbf{M}$ refine the value $\perp$, and are helpful for falsification and testing, as they indicate that the abstract state corresponds to at least one concrete state that satisfies the property $\left(\mathbf{T}_{\exists}\right)$, at least one concrete state that violates the property $\left(\mathbf{F}_{\exists}\right)$, and at least one pair of concrete states in which one state satisfies the property, and the other state violates it (M).

As shown in the figure below, the six values $\mathcal{L}_{6}=\left\{\mathbf{T}, \mathbf{F}, \mathbf{M}, \mathbf{T}_{\exists}, \mathbf{F}_{\exists}, \perp\right\}$ can be ordered in the information lattice depicted on the left. The values can also be ordered in the "truth lattice" depicted on the right:

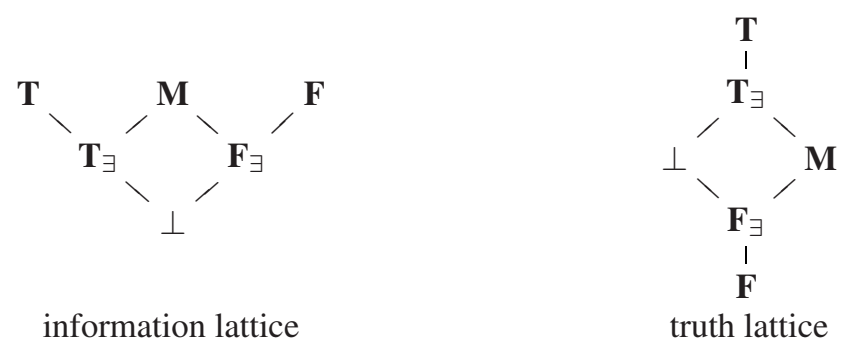

We allow the truth values of the (abstract) labeling function $L_{A}$ to range over the six truth values.

A TMTS $A=\left\langle A P, S_{A}, I_{A}, \stackrel{\text { may }}{\longrightarrow}, \stackrel{\text { must }^{+}}{\longrightarrow}, \stackrel{\text { must }}{-}_{A}, L_{A}\right\rangle$ is an abstraction of a concrete transition system $C=\left\langle A P, S_{C}, I_{C}, \longrightarrow_{C}, L_{C}\right\rangle$ if there exists a total and onto function $\rho: S_{C} \rightarrow S_{A}$ such that for all $a \in S_{A}$ and $p \in A P$ :

- $L_{A}(a, p)=\mathbf{T}$ only if for all $c \in S_{C}$ such that $\rho(c)=a, L_{C}(c, p)=\mathbf{T}$;

- $L_{A}(a, p)=\mathbf{F}$ only if for all $c \in S_{C}$ such that $\rho(c)=a, L_{C}(c, p)=\mathbf{F}$;

- $L_{A}(a, p)=\mathbf{T}_{\exists}$ only if there exists $c \in S_{C}$ such that $\rho(c)=a$ and $L_{C}(c, p)=\mathbf{T}$;

- $L_{A}(a, p)=\mathbf{F}_{\exists}$ only if there exists $c \in S_{C}$ such that $\rho(c)=a$ and $L_{C}(c, p)=\mathbf{F}$;

- $L_{A}(a, p)=\mathbf{M}$ only if there exist $c, c^{\prime} \in S_{C}$ such that $\rho(c)=\rho\left(c^{\prime}\right)=a$, $L_{C}(c, p)=\mathbf{T}$, and $L_{C}\left(c^{\prime}, p\right)=\mathbf{F}$.

In addition, $\rho$ satisfies the requirement (ii) defined in Section 2.1.

\footnotetext{
${ }^{5}$ The specifications may contain both future and past operators. For simplicity, we describe the framework here for the $\mu$-calculus, which does not contain past modalities. By letting the $A Y$ modality range over must ${ }^{+}$transitions, the framework can be used for falsification of full $\mu$-calculus specifications.
} 
The complementation $\left(\neg: \mathcal{L}_{6} \rightarrow \mathcal{L}_{6}\right)$ and the conjunction $\left(\wedge: \mathcal{L}_{6} \times \mathcal{L}_{6} \rightarrow \mathcal{L}_{6}\right)$ operations are defined as follows:

\begin{tabular}{|c|c|c|c|}
\hline & $\neg$ & $\wedge$ & $\mid \mathbf{F} \mathbf{F}_{\exists} \mathbf{M} \mathbf{T}_{\exists} \mathbf{T} \perp$ \\
\hline $\mathbf{F}$ & $\mathbf{T}$ & $\mathbf{F}$ & \begin{tabular}{|llllll}
$\mathbf{F}$ & $\mathbf{F}$ & $\mathbf{F}$ & $\mathbf{F}$ & $\mathbf{F}$ & $\mathbf{F}$
\end{tabular} \\
\hline $\mathbf{F}_{\exists}$ & $\mathbf{T}_{\exists}$ & $\mathbf{F}_{\exists}$ & $\mathbf{F} \mathbf{F}_{\exists} \mathbf{F}_{\exists} \mathbf{F}_{\exists} \mathbf{F}_{\exists} \mathbf{F}_{\exists}$ \\
\hline $\mathbf{M}$ & M & $\mathbf{M}$ & $\mathbf{F} \mathbf{F}_{\exists} \mathbf{F}_{\exists} \mathbf{F}_{\exists} \mathbf{M} \mathbf{F}_{\exists}$ \\
\hline $\mathbf{T}_{\exists}$ & $\mathbf{F}_{\exists}$ & $\mathbf{T}_{\exists}$ & $\mathbf{F} \mathbf{F}_{\exists} \mathbf{F}_{\exists} \perp \mathbf{T}_{\exists} \perp$ \\
\hline $\mathbf{T}$ & $\mathbf{F}$ & $\mathbf{T}$ & $\mathbf{F} \mathbf{F}_{\exists} \mathbf{M} \mathbf{T}_{\exists} \mathbf{T} \perp$ \\
\hline$\perp$ & $\perp$ & $\perp$ & $\mathbf{F} \mathbf{F}_{\exists} \mathbf{F}_{\exists} \perp \perp \perp$ \\
\hline
\end{tabular}

We define a 6-valued falsification semantics of PML formulas with respect to TMTS. The value of a formula $\theta$ in a state $a$ of a TMTS $A=\left\langle A P, S_{A}, I_{A}, \stackrel{\text { may }}{\longrightarrow}_{A}, \stackrel{\text { must }}{+}_{A}, \stackrel{\text { must }}{\longrightarrow} A\right.$, $\left.L_{A}\right\rangle$, denoted $[(A, a) \mid=\theta]$, is defined as follows:

$$
\begin{aligned}
& {[(A, a) \models p]=L_{A}(a, p) .} \\
& {[(A, a) \models \neg \theta]=\neg([(A, a) \models \theta])} \\
& {\left[(A, a) \models \theta_{1} \wedge \theta_{2}\right]=\wedge\left(\left[\left(A, a^{\prime}\right) \models \theta_{1}\right],\left[\left(A, a^{\prime}\right) \models \theta_{2}\right]\right)} \\
& {[(A, a) \models A X \theta]= \begin{cases}\mathbf{T} \text { if for all } a^{\prime}, \text { if } \operatorname{may}\left(a, a^{\prime}\right) \text { then }\left[\left(A, a^{\prime}\right) \models \theta\right]=\mathbf{T} . \\
\mathbf{F} \text { if exists } a^{\prime} \text { s.t. must }{ }^{+}\left(a, a^{\prime}\right) \text { and }\left[\left(A, a^{\prime}\right) \models \theta\right]=\mathbf{F} . \\
\mathbf{F}_{\exists} \text { if exists } a^{\prime} \text { s.t. must }\left(a, a^{\prime}\right) \text { and }\left[\left(A, a^{\prime}\right) \models \theta\right] \sqsupseteq \mathbf{F}_{\exists} . \\
\perp \text { otherwise. }\end{cases} }
\end{aligned}
$$

Note that the conditions for the $\mathbf{F}$ and the $\mathbf{F}_{\exists}$ conditions are not mutually exclusive. If both conditions hold, we take the value to be the stronger $\mathbf{F}$ value.

For clarity, we give the semantics for the existential operator $E X$ explicitly (an equivalent definition follows from the semantics of $A X$ and $\neg$ ):

$$
[(A, a) \models E X \theta]=\left\{\begin{array}{l}
\mathbf{F} \quad \text { if for all } a^{\prime} \text {, if } \operatorname{may}\left(a, a^{\prime}\right) \text { then }\left[\left(A, a^{\prime}\right) \models \theta\right]=\mathbf{F} . \\
\mathbf{T} \quad \text { if exists } a^{\prime} \text { s.t. } \operatorname{must}^{+}\left(a, a^{\prime}\right) \text { and }\left[\left(A, a^{\prime}\right) \models \theta\right]=\mathbf{T} . \\
\mathbf{T}_{\exists} \text { if exists } a^{\prime} \text { s.t. } \operatorname{must}^{-}\left(a, a^{\prime}\right) \text { and }\left[\left(A, a^{\prime}\right) \models \theta\right] \sqsupseteq \mathbf{T}_{\exists} . \\
\perp \text { otherwise. }
\end{array}\right.
$$

Thus, the semantics of the next-time operators follows both must $^{-}$and must $^{+}$ transitions (that is, $a^{\prime}$ is such that must $^{-}\left(a, a^{\prime}\right)$ or must $\left.^{+}\left(a, a^{\prime}\right)\right)$. To understand why must $^{-}$transitions are suitable for falsification, let us explain the positive falsification semantics for the $E X$ modality. The other cases are similar. Consider a concrete transition system $C=\left\langle A P, S_{C}, I_{C}, \longrightarrow_{C}, L_{C}\right\rangle$, and an abstraction for it $A=$ $\left\langle A P, S_{A}, I_{A}, \stackrel{\text { may }}{\longrightarrow}_{A}, \stackrel{\text { must }}{+}_{A}, \stackrel{\text { must }}{ }_{A}^{-}, L_{A}\right\rangle$. Let $\rho: S_{C} \rightarrow S_{A}$ be the witness function for the abstraction.

We argue that if $[(A, a) \models E X p]=\mathbf{T}_{\exists}$, then there is a concrete state $c$ such that $\rho(c)=a$ and $c \models E X p$. By the semantics of the $E X$ operator, $[(A, a) \models E X p]=\mathbf{T}_{\exists}$ implies that there is $a^{\prime} \in S_{A}$ such that $\operatorname{must}^{-}\left(a, a^{\prime}\right)$ and $L_{A}\left(a^{\prime}, p\right) \sqsupseteq \mathbf{T}_{\exists}$. Let $\hat{c}$ be a concrete state with $\rho(\hat{c})=a^{\prime}$ and $L_{C}(\hat{c}, p)=\mathbf{T}$ (by the definition of abstraction, at least one such $\hat{c}$ exists). Since must ${ }^{-}\left(a, a^{\prime}\right)$, then for every concrete state $c^{\prime}$ such that $\rho\left(c^{\prime}\right)=a^{\prime}$ there is a concrete state $c$ such that $\rho(c)=a$ and $c \longrightarrow C c^{\prime}$. In particular, there is a concrete state $c$ such that $\rho(c)=a$ and $c \longrightarrow_{C} \hat{c}$. Thus, $c=E X p$ and we are done. 
Let $a$ and $a^{\prime}$ be abstract states. The (reflexive) transitive closure of must $^{-}$, denoted $\left[\text { must }^{-}\right]^{*}$ is defined in the expected manner as follows: $\left[\text { must }^{-}\right]^{*}\left(a, a^{\prime \prime}\right)$ if either $a=$ $a^{\prime \prime}$ or there is an abstract state $a^{\prime}$ such that $\left[\text { must }^{-}\right]^{*}\left(a, a^{\prime}\right)$ and must $^{-}\left(a^{\prime}, a^{\prime \prime}\right)$. We say that an abstract state $a^{\prime}$ is onto reachable from an abstract state $a$ if for every concrete state $c^{\prime}$ that satisfies $a^{\prime}$, there is a concrete state $c$ that satisfies $a$ and $c^{\prime}$ is reachable from c. Dually, we can define the transitive closure of must $^{+}$transitions, denoted $\left[\text {must }^{+}\right]^{*}$, and total reachability. The transitive closure of must $^{+}$and must $^{-}$transitions retain the reachability properties for a single transition: $\left[\mathrm{must}^{-}\right]^{*}\left(a, a^{\prime}\right)$ only if $a^{\prime}$ is onto reachable from $a$, and $\left[\mathrm{must}^{+}\right]^{*}\left(a, a^{\prime}\right)$ only if $a^{\prime}$ is total reachable from $a$ [Bal04].

By extending PML by fixed-point operators, one gets the logic $\mu$-calculus [Koz83], which subsumes the branching temporal logics CTL and CTL ${ }^{\star}$. The 3-valued semantics of PML can be extended to the $\mu$-calculus [BG04]. Note that in the special case of CTL and $\mathrm{CTL}^{\star}$ formulas, this amounts to letting path formulas range over may and must ${ }^{+}$paths [SG03]. The fact that the "onto" nature of must $^{-}$transitions is retained under transition closure enables us to extend the soundness argument for the 6 -valued falsification semantics described above for a single $E X$ or $A X$ modality to nesting of such modalities and thus, to PML and the $\mu$-calculus, as shown in our technical report [BKY05].

\section{Weak Reachability}

When reasoning about paths in the abstract system, one can often manage with an even weaker type of reachability (than transitive closure over must $^{-}$transitions): we say that an abstract state $a^{\prime}$ is weakly reachable from an abstract state $a$ if there is a concrete state $c^{\prime}$ that satisfies $a^{\prime}$, there is a concrete state $c$ that satisfies $a$, and $c^{\prime}$ is reachable from $c$. The combination of must ${ }^{+}$and must $^{-}$transitions turn out to be especially powerful when reasoning about weak reachability.

If there are three abstract states $a_{1}, a_{2}$, and $a_{3}$ such that $a_{2}$ is onto reachable from $a_{1}$ and $a_{3}$ is total reachable from $a_{2}$, then $a_{3}$ is weakly reachable from $a_{1}$. Hence, weak reachability can be concluded from the existence of a sequence of must $^{-}$transitions followed immediately by a sequence of must $^{+}$transitions:

Theorem 2. [Bal04] If $\left[\text { must }^{-}\right]^{*}\left(a_{1}, a_{2}\right)$ and $\left[\text { must }^{+}\right]^{*}\left(a_{2}, a_{3}\right)$, then $a_{3}$ is weakly reachable from $a_{1}$.

\subsection{Weak Reachability in Predicate Abstraction}

We now focus on the case where the concrete system is a program, and its abstraction is obtained by predicate abstraction. We then show that weak reachability can be made tighter by parameterizing the abstract transitions by predicates. The predicates used in these transitions may be (and usually are) different from the predicates used for predicate abstraction.

Consider a program $P$. Let $X$ be the set of variables appearing in the program and variables that encode the program location, and let $D$ be the domain of all variables (for technical simplicity, we assume that all variables are over the same domain). We model $P$ by a concrete transition system in which each state is labeled by a valuation in $D^{X}$. 


\begin{tabular}{|lc|}
\hline L0 & if $x<6$ then \\
L1 & $x:=x+3$ \\
L2 & if $x>7$ then \\
L3 & $x:=x-3$ \\
L4 & end
\end{tabular}

Fig. 1. The program $P$

Let $\Phi=\left\{\phi_{1}, \phi_{2}, \ldots, \phi_{n}\right\}$ be a set of predicates (quantifier-free formulas of first-order logic) on $X$. For a set $a \subseteq \Phi$ and an assignment $c \in D^{X}$, we say that $c$ satisfies $a$ iff $c$ satisfies all the predicates in $a$. The satisfaction relation induces a total and onto function $\rho: D^{X} \rightarrow 2^{\Phi}$, where $\rho(c)=a$ for the unique $a$ for which $c$ satisfies $a$. An abstraction of the program $P$ that is based on $\Phi$ is a TMTS with state space $2^{\Phi}$, thus each state is associated (and is labeled by) the set of predicates that hold in it. For a detailed description of predicate abstraction see [GS97].

Note that all the transitions of the concrete system in which only the variables that encode the program location are changed (all transitions associated with statements that are not assignments, c.f., conditional branches, skip, etc.) are both must $^{+}$and $m u s t^{-}$ transitions, assuming that $\Phi$ includes all conditional expressions in the program. We call such transitions silent transitions. The identification of silent transitions makes our reasoning tighter: if $a \stackrel{\text { silent }}{\longrightarrow} a^{\prime}$ we can replace the transition from $a$ to $a^{\prime}$ with transitions from $a$ 's predecessors to $a^{\prime}$. The type of a new transition is the same as the type of the transitions leading to $a .{ }^{6}$ Such elimination of silent transitions result in an abstract system in which each transition is associated with an assignment statement.

For simplicity of exposition, we present a toy example. ${ }^{7}$ Consider the program $P$ appearing in Figure 1.

When describing an abstract system, it is convenient to describe an abstract state in $S_{A}$ as a pair of program location and a Boolean vector describing which of the program predicates in $\Phi$ hold. Let $\phi_{1}=(x<6)$ and $\phi_{2}=(x>7)$. The abstraction of $P$ that corresponds to the two predicates is described in the left-hand side of Figure 2. In the right-hand side, we eliminate the silent transitions.

We now turn to study weak reachability in the abstract system. By Theorem 2, if $\left[\text { must }^{-}\right]^{*}\left(a_{1}, a_{2}\right)$ and $\left[\text { must }^{+}\right]^{*}\left(a_{2}, a_{3}\right)$, then $a_{3}$ is weakly reachable from $a_{1}$. While Theorem 2 is sound, it is not complete, in the sense that it is possible to have two abstract states $a$ and $a^{\prime}$ such that $a^{\prime}$ is weakly reachable from $a$ and still no sequence of transitions as specified in Theorem 2 exists in the abstract system. As an example, consider the abstract states $a=(\mathrm{L} 1: \mathrm{TF})$ and $a^{\prime}=(\mathrm{L} 4: \mathrm{TF})$. While $a^{\prime}$ is weakly reachable from $a$; c.f., $c^{\prime}=(\mathrm{L} 4: x=5)$ is reachable from $c=(\mathrm{L} 0: x=5)$, the only path from $a$ to $a^{\prime}$ in the abstraction contains two may transitions, so Theorem 2 cannot be

\footnotetext{
${ }^{6}$ A transition from $a^{\prime}$ may also be silent, in which case we continue until the chain of silent transitions either reaches an end state or reaches an assignment statement. If the chain reaches an end state, we can make $a$ an end state.

${ }^{7}$ Our ideas have proven useful also in real examples, as described in our technical report [BKY05].
} 


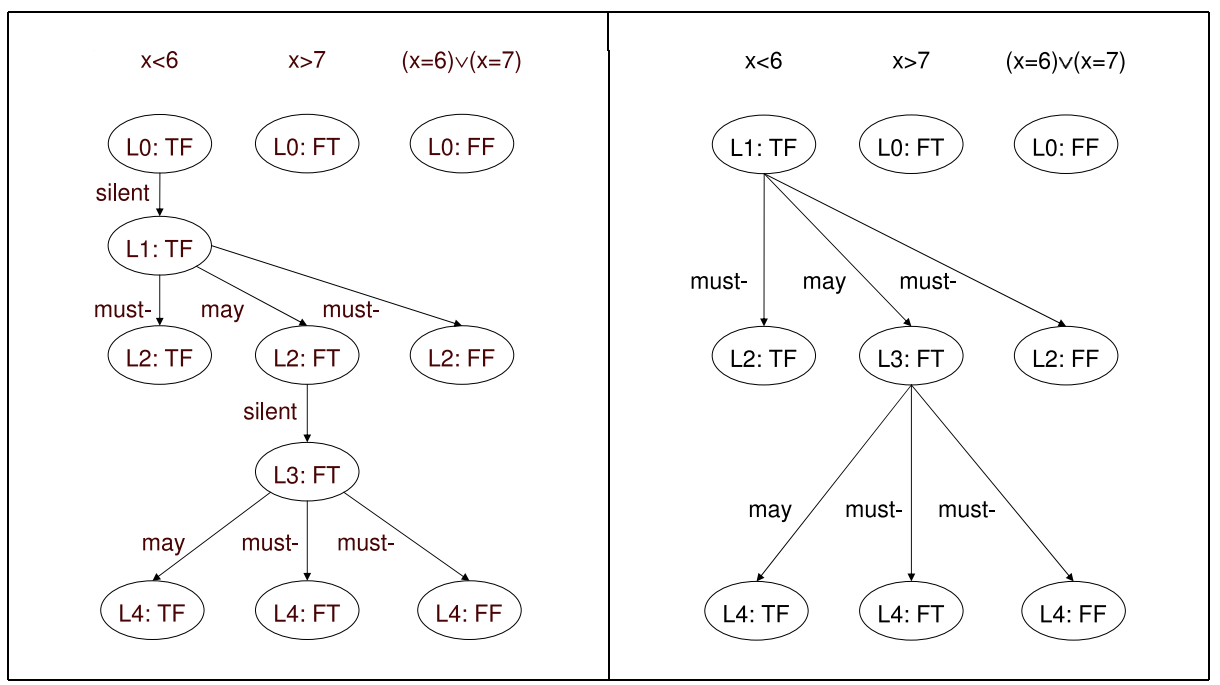

Fig. 2. The abstract transition system of the program $P$ from Figure 1

applied. In fact, the status of the abstract states (L4:FT) and (L4:FF) also is not clear, as the paths from $a$ to these states do not follow the sequence specified in Theorem 2 . Accordingly, Theorem 2 does not help us determining whether there is an input $x<6$ to $P$ such that the execution of $P$ on $x$ would reach location L4 with $x$ that is strictly bigger than 7 or with $x$ that is equal to 6 or 7 . Our goal is to tighten Theorem 2 , so that we end up with fewer such undetermined cases.

\subsection{Parameterized Must Transitions}

Recall that each abstract state is associated with a location of the program, and thus it is also associated with a statement. For a statement $s$ and a predicate $e$ over $X$, the weakest precondition $\mathrm{WP}(s, e)$ and the strongest postcondition $\operatorname{SP}(s, e)$ are defined as follows [Dij76]:

- The execution of $s$ from every state that satisfies $\operatorname{WP}(s, e)$ results in a state that satisfies $e$, and $\operatorname{WP}(s, e)$ is the weakest predicate for which the above holds.

- The execution of $s$ from a state that satisfies $e$ results in a state that satisfies $\operatorname{SP}(s, e)$, and $\operatorname{SP}(s, e)$ is the strongest predicate for which the above holds.

For example, in the program $P$, we have $\mathrm{WP}(x:=x+3, x>7)=x>4$, $\mathrm{SP}(x:=x+3, x<6)=x<9, \mathrm{WP}(x:=x-3, x<6)=x<9$, and $\operatorname{SP}(x:=$ $x-3, x>7)=x>4$.

Let $\theta$ be a predicate over $X$. We parameterize must $^{+}$and must $^{-}$transitions by $\theta$ as follows:

- ust $^{+}(\theta)\left(a, a^{\prime}\right)$ only if for every concrete state $c$ that satisfies $a \wedge \theta$, there is a concrete state $c^{\prime}$ that satisfies $a^{\prime}$ and $c \longrightarrow_{C} c^{\prime}$.

- must ${ }^{-}(\theta)\left(a, a^{\prime}\right)$ only if for every concrete state $c^{\prime}$ that satisfies $a^{\prime} \wedge \theta$, there is a concrete state $c$ that satisfies $a$ and $c \longrightarrow_{C} c^{\prime}$. 
Thus, a must ${ }^{+}(\theta)$ transition is total from all states that satisfy $\theta$, and a ust $^{-}(\theta)$ transition is onto all states that satisfy $\theta$. Note that when $\theta=\mathbf{T}$, we get usual must ${ }^{+}$ and must $^{-}$transitions. Parameterized transitions can be generated automatically (using WP and SP) while building the TMTS without changing the complexity of the abstraction algorithm.

Theorem 3. Let $a$ and $a^{\prime}$ be two abstract states, and $s$ the statement executed in a. Then, must ${ }^{+}\left(\mathrm{WP}\left(s, a^{\prime}\right)\right)\left(a, a^{\prime}\right)$ and must ${ }^{-}(\operatorname{SP}(s, a))\left(a, a^{\prime}\right)$.

The good news about Theorem 3 is that it is complete in the sense that for all predicates $\theta$, if there is a must $t^{+}(\theta)$ transition from $a$ to $a^{\prime}$, then $a \rightarrow\left(\theta \rightarrow \mathrm{WP}\left(s, a^{\prime}\right)\right)$, and similarly for must $^{-}$transitions, as formalized below.

Lemma 1. Let a and $a^{\prime}$ be two abstract states, and $s$ the statement executed in a.

- If there is a must ${ }^{+}(\theta)$ transition from a to $a^{\prime}$, then $a \rightarrow\left(\theta \rightarrow \operatorname{WP}\left(s, a^{\prime}\right)\right)$.

- If there is a must ${ }^{-}(\theta)$ transition from a to $a^{\prime}$, then $a^{\prime} \rightarrow(\theta \rightarrow \operatorname{SP}(s, a))$.

Thus, the pre and post conditions, which can be generated automatically, are the strongest predicates that can be used. Note that using Theorem 3, it is possible to replace all may transitions by parameterized must $^{-}$and must $^{+}$transitions.

It is easy to see how parameterized transitions can help when we consider weak reachability. Indeed, if must ${ }^{-}\left(\theta_{1}\right)\left(a, a^{\prime}\right)$, must $^{+}\left(\theta_{2}\right)\left(a^{\prime}, a^{\prime \prime}\right)$, and $\theta_{1} \wedge \theta_{2} \wedge a^{\prime}$ is satisfiable, then $a^{\prime \prime}$ is weakly reachable from $a$, as formalized by the following lemma.

Lemma 2. If must ${ }^{-}\left(\theta_{1}\right)\left(a, a^{\prime}\right)$, must ${ }^{+}\left(\theta_{2}\right)\left(a^{\prime}, a^{\prime \prime}\right)$, and $\theta_{1} \wedge \theta_{2} \wedge a^{\prime}$ is satisfiable, then there are concrete states $c$ and $c^{\prime \prime}$ such that $a(c), a^{\prime \prime}\left(c^{\prime \prime}\right)$, and $c^{\prime \prime}$ is reachable from $c$.

The completeness of Theorem 3 implies that when $a^{\prime}$ is weakly reachable from $a$ via two transitions, this always can be detected by taking $\theta_{1}=\operatorname{SP}(s, a)$ and $\theta_{2}=$ $\mathrm{WP}\left(s^{\prime}, a^{\prime}\right)$, where $s$ and $s^{\prime}$ are the statements executed in the two transitions.

In our example, we have seen that the transitions from (L1:TF) to (L3:FT) and from (L3:FT) to (L4:TF) are both may transitions, and thus Theorem 2 cannot be applied. However, the fact that the first transition also is a must $^{-}(x<9)$ transition and the second also is a must ${ }^{+}(x<9)$, together with the fact that $x>7 \wedge x<9$ is satisfiable, guarantee that there is a concrete state that corresponds to (L1:TF) and from which a concrete state that corresponds to (L4:TF) is reachable. Indeed, as we noted earlier, $(\mathrm{L} 4: x=5)$ is reachable from $(\mathrm{LO}: x=5)$.

When $a$ and $a^{\prime}$ are of distance greater than two transitions, parameterization is useful for composing the sequence of must $^{-}$transitions with the sequence of must $^{+}$ transitions:

Theorem 4. If $\left[\text { must }^{-}\right]^{*}\left(a_{1}, a_{2}\right)$, must ${ }^{-}\left(\theta_{1}\right)\left(a_{2}, a_{3}\right)$, must $^{+}\left(\theta_{2}\right)\left(a_{3}, a_{4}\right),\left[\text { must }^{+}\right]^{*}$ $\left(a_{4}, a_{5}\right)$, and $a_{3} \wedge \theta_{1} \wedge \theta_{2}$ is satisfiable, then $a_{5}$ is weakly reachable from $a_{1}$.

Again, the predicates $\theta_{1}$ and $\theta_{2}$ are induced by the pre and postconditions of the statement leading to the abstract state in which the two sequences are composed. 
The transitive closure of the parameterized must transitions does not retain the reachability properties of a single transition and requires reasoning in an assume-guarantee fashion, where two predicates are associated with each transition. Our technical report [BKY05] presents such an extension and shows how to use it to extend the set of reachable states further.

\section{Applications}

This section describes application of weak reachability for linear-time falsification and for abstraction-guided test generation.

In linear-time model checking, we check whether all the computations of a given program $P$ satisfy a specification $\psi$, say an LTL formula. In the automata-theoretic approach to model checking, one constructs an automaton $\mathcal{A}_{\neg \psi}$ for the negation of $\psi$. The automaton $\mathcal{A}_{\neg \psi}$ is usually a nondeterministic Büchi automaton, where a run is accepting iff it visits a set of designated states infinitely often. The program $P$ is faulty with respect to $\psi$ if the product of $\mathcal{A}_{\neg \psi}$ with the program contains a fair path one that visits the set of designated states infinitely often. The product of $\mathcal{A}_{\neg \psi}$ with an abstraction of $P$ may contain fair paths that do not correspond to computations of $P$, thus again there is a need to check for weak reachability.

When reasoning about concrete systems, emptiness of the product automaton can be reduced to a search for an accepting state that is reachable from both an initial state and itself. In the context of abstraction, we should make sure that the path from the accepting state to itself can be repeated, thus weak reachability is too weak here ${ }^{8}$, and instead we need the following.

Theorem 5. If, in the product automaton of $P$ with respect to LTL formula $\psi$, there is an initial abstract state $a_{\text {init }}$ and an accepting state $a_{\text {acc }}$ such that $a_{\text {acc }}$ is onto reachable from $a_{\text {init }}$ and from itself, or $a_{a c c}$ is weakly reachable from $a_{\text {init }}$ and total reachable from itself, then $P$ violates $\psi$.

Falsification methods are related to testing, where the system is actually executed. The infeasible task of executing the system with respect to all inputs is replaced by checking a test suite consisting of a finite subset of inputs. It is very important to measure the exhaustiveness of the test suite, and indeed, there has been an extensive research in the testing community on coverage metrics, which provide such a measure.

Some coverage metrics are defined with respect to an abstraction of the system. For example, in predicate-complete testing [Bal04], the goal is to cover all the reachable observable states (evaluation of the system's predicates under all reachable states), and reachability is studied in an abstract system whose state space consists of an overapproximation of the reachable observable states. The observable states we want our test suite to cover are abstract states that are weakly reachable.

The fundamental question in this setting is how to determine which abstract states are weakly reachable. As we have seen, TMTS provide a sufficient condition for deter-

\footnotetext{
${ }^{8}$ When $\psi$ is a safety property, $\mathcal{A}_{\neg \psi}$ is an automaton accepting finite bad prefixes [KV01], and weak reachability is sufficient.
} 
mining weak reachability (via a sequence of must $^{-}$transitions followed by a sequence of must $^{+}$transitions). The parameterization method makes this condition tighter.

\section{Conclusion}

We have described an abstraction framework that contains must $^{-}$transitions, the backwards version of must transitions, and showed how must ${ }^{-}$transitions enable reasoning about past-time modalities as well as future-time modalities in a falsification semantics. We showed that the falsification setting allows for a stronger type of abstraction and described applications in falsification of temporal properties and testing.

A general idea in our work is that by replacing must $^{+}$by must $^{-}$transitions, abstraction frameworks that are sound for verification become abstraction frameworks that are sound (and more precise) for falsification. We demonstrated it with model checking and refinement, and we believe that several other ideas in verification can be lifted to falsification in the same way. This includes generalized model checking [GJ02], making the framework complete [DN05], and its augmentation with hyper-transitions [LX90, SG04].

Another interesting direction is to use must $^{-}$transitions in order to strengthen abstractions in the verification setting: the ability to move both forward and backwards across the transition relation has proven helpful in the concrete setting. Using must ${ }^{-}$ transitions, this also can be done in the abstraction setting.

\section{References}

[Bal04] T. Ball. A theory of predicate-complete test coverage and generation. In 3rd International Symposium on Formal Methods for Components and Objects, 2004.

[Ben91] J. Benthem. Languages in actions: categories, lambdas and dynamic logic. Studies in Logic, 130, 1991.

[BG99] G. Bruns and P. Godefroid. Model checking partial state spaces with 3-valued temporal logics. In Computer Aided Verification, pages 274-287, 1999.

[BG04] G. Bruns and P. Godefroid. Model checking with 3-valued temporal logics. In 31 st International Colloquium on Automata, Languages and Programming, volume 3142 of Lecture Notes in Computer Science, pages 281-293, 2004.

[BKY05] T. Ball, O. Kupferman, and G. Yorsh. Abstraction for falsification. Technical Report MSR-TR-2005-50, Microsoft Research, 2005.

[CC77] P. Cousot and R. Cousot. Abstract interpretation: a unified lattice model for the static analysis of programs by construction or approximation of fixpoints. In $P O P L$ 77: Principles of Programming Languages, pages 238-252. ACM, 1977.

[CE81] E.M. Clarke and E.A. Emerson. Design and synthesis of synchronization skeletons using branching time temporal logic. In Proc. Workshop on Logic of Programs, volume 131 of Lecture Notes in Computer Science, pages 52-71. Springer-Verlag, 1981.

[DGG97] D. Dams, R. Gerth, and O. Grumberg. Abstract interpretation of reactive systems. ACM Trans. Program. Lang. Syst., 19(2):253-291, 1997.

[Dij76] E.W. Dijksta. A Discipline of Programming. Prentice-Hall, 1976. 
[DN05] D. Dams and K. S. Namjoshi. Automata as abstractions. In VMCAI 2005, Paris, 2005. to appear, LNCS, Springer-Verlag.

$\left[\mathrm{FKZ}^{+} 00\right] \quad$ R. Fraer, G. Kamhi, B. Ziv, M. Vardi, and L. Fix. Prioritized traversal: efficient reachability analysis for verication and falsification. In Proc. 12th Conference on Computer Aided Verication, volume 1855 of Lecture Notes in Computer Science, pages 389-402, Chicago, IL, USA, July 2000. Springer-Verlag.

[GHJ01] P. Godefroid, M. Huth, and R. Jagadeesan. Abstraction-based model checking using modal transition systems. In Proceedings of CONCUR'2001 (12th International Conference on Concurrency Theory), volume 2154 of Lecture Notes in Computer Science, pages 426-440. Springer-Verlag, 2001.

[GJ02] P. Godefroid and R. Jagadeesan. Automatic abstraction using generalized model checking. In Computer Aided Verification, pages 137-150, 2002.

[GLST05] O. Grumberg, F. Lerda, O. Strichman, and M. Theobald. Proof-guided underapproximation-widening for multi-process systems. In POPL, pages 122$131,2005$.

[GS97] S. Graf and H. Saidi. Construction of abstract state graphs with PVS. In CAV 97: Computer-aided Verification, LNCS 1254, pages 72-83. Springer-Verlag, 1997.

[HJS01] M. Huth, R. Jagadeesan, and D. Schmidt. Model checking partial state spaces with 3-valued temporal logics. In ESOP, pages 155-169, 2001.

[Kle87] S. C. Kleene. Introduction to Metamathematics. North Holland, 1987.

[Koz83] D. Kozen. Results on the propositional $\mu$-calculus. Theoretical Computer Science, 27:333-354, 1983.

[KV01] O. Kupferman and M.Y. Vardi. Model checking of safety properties. Formal methods in System Design, 19(3):291-314, November 2001.

[LT88] K.G. Larsen and G.B. Thomsen. A modal process logic. In Proc. 3th Symp. on Logic in Computer Science, Edinburgh, 1988.

[LX90] K. G. Larsen and L. Xinxin. Equation solving using modal transition systems. In LICS, pages 108-117, 1990.

[PDV01] C. S. Pasareanu, M. B. Dwyer, and W. Visser. Finding feasible counter-examples when model checking abstracted java programs. In TACAS, pages 284-298, 2001.

[QS81] J.P. Queille and J. Sifakis. Specification and verification of concurrent systems in Cesar. In Proc. 5th International Symp. on Programming, volume 137 of Lecture Notes in Computer Science, pages 337-351. Springer-Verlag, 1981.

[SG03] S. Shoham and O. Grumberg. A game-based framework for CTL counterexamples and 3-valued abstraction-refinement. In Computer Aided Verification, pages 275287, 2003.

[SG04] S. Shoham and O. Grumberg. Monotonic abstraction-refinement for CTL. In Tools and Algorithms for Construction and Analysis of Systems (TACAS), volume 2988 of Lecture Notes in Computer Science, pages 546-560. Springer-Verlag, 2004.

[Sip99] H.B. Sipma. Diagram-based Verification of Discrete, Real-time and Hybrid Systems. PhD thesis, Stanford University, Stanford, California, 1999. 